Bull. Soc. math. France

129 (2), 2001, p. 169-174

\title{
GALOIS-FIXED POINTS IN THE BRUHAT-TITS BUILDING OF A REDUCTIVE GROUP
}

\author{
By Gopal Prasad
}

\begin{abstract}
We give a new proof of a useful result of Guy Rousseau on Galois-fixed points in the Bruhat-Tits building of a reductive group.

RÉSumÉ (Points fixes de Galois dans l'immeuble de Bruhat-Tits d'un groupe réductif)

Nous donnons une nouvelle preuve d'un résultat utile de Guy Rousseau sur les points fixes de Galois dans l'immeuble de Bruhat-Tits d'un groupe réductif.
\end{abstract}

Let $k$ be a field with a nontrivial discrete valuation. We assume that $k$ is complete and its residue field is perfect. Let $p(\geq 0)$ be the characteristic of the residue field. Let $G$ be an absolutely almost simple simply connected algebraic group defined over $k$. The Bruhat-Tits building $\mathcal{B}(G / \ell)$ of $G / \ell$ exists for any algebraic extension $\ell$ of $k$ and it is functorial in $\ell$ (see [2, $\S 5]$ or [4]). If $\ell$ is a Galois extension of $k$, there is a natural action, by simplicial isometries, of the Galois group $\operatorname{Gal}(\ell / k)$ on the building $\mathcal{B}(G / \ell)$ (see [2, 4.2.12], or [4, Chap. II]). The convex subset consisting of points of $\mathcal{B}(G / \ell)$ fixed under $\operatorname{Gal}(\ell / k)$ will be denoted by $\mathcal{B}(G / \ell)^{\operatorname{Gal}(\ell / k)} ; \mathcal{B}(G / \ell)^{\operatorname{Gal}(\ell / k)}$ contains $\mathcal{B}(G / k)$. It is known (and, in fact, this result is an important component of the Bruhat-Tits theory) that if $\ell$ is an unramified extension of $k$, then $\mathcal{B}(G / \ell)^{\operatorname{Gal}(\ell / k)}$ coincides with $\mathcal{B}(G / k)$, see $[2,5.1 .25]$. However, in general, the former is larger than $\mathcal{B}(G / k)$ (see [8, 2.6.1]). Guy Rousseau in his unpublished thesis [4] proved that if $\ell$ is a

Texte reçu le 4 janvier 2000, révisé le 8 mars 2000

Gopal Prasad, Department of Mathematics, University of Michigan, Ann Arbor MI 481091109 (USA) • E-mail : gprasad@math.lsa.umich.edu

Key words and phrases. - Reductive group, Bruhat-Tits building, Galois fixed points.

Partially supported by a Guggenheim Foundation Fellowship, a BSF (Israel-US) grant and a NSF-grant. 
tamely ramified finite Galois extension of $k$, then again $\mathcal{B}(G / \ell)^{\operatorname{Gal}(\ell / k)}$ coincides with $\mathcal{B}(G / k)$. This result has recently been used in the representation theory of, and harmonic analysis on, $G(k)$. The purpose of this note is to provide a short proof of the result.

Let $\mathfrak{K}$ be a field with a nontrivial discrete valuation and containing $k$ as a valuated subfield. We assume that $\mathfrak{K}$ is henselian with respect to the given valuation and its residue field is perfect. Then $G$ admits the Bruhat-Tits building $\mathcal{B}(G / \mathfrak{K})$ over $\mathfrak{K}$; see $[2, \S 5]$. Let $\widehat{\mathfrak{K}}$ be the completion of $\mathfrak{K}$. Using the following version of Hensel's lemma: for any smooth variety $V$ defined over $\mathfrak{K}, V(\mathfrak{K})$ is dense in $V(\widehat{\mathfrak{K}})$ in the topology on the latter induced by the topology on $\widehat{\mathfrak{K}}$, Bruhat, Tits and Rousseau have shown $([4, \mathrm{II}, \S 3])$ that $\mathfrak{K}$-rank $G=\widehat{\mathfrak{K}}$-rank $G$, and the Bruhat-Tits building $\mathcal{B}(G / \widehat{\mathfrak{K}})$ of $G / \widehat{\mathfrak{K}}$ is equal to the building $\mathcal{B}(G / \mathfrak{K})$.

Let $K$ be the completion of a fixed maximal unramified extension of $k$. Let $L$ be a finite tamely ramified Galois extension of $K$ and $\Gamma=\operatorname{Gal}(L / K)$. In view of the results of Bruhat and Tits, and of Bruhat, Tits and Rousseau mentioned above, to establish the theorem of Rousseau, it suffices to show that

$$
\mathcal{B}(G / L)^{\Gamma}=\mathcal{B}(G / K) .
$$

This is what we will do below.

Let $S$ be a maximal $K$-split torus of $G$. It is a well known consequence of a theorem of Steinberg (see $[6],[1,8.6]$ ) that $G$ is quasi-split over $K$, i.e. it contains a Borel subgroup defined over $K$. Hence, the centralizer $\mathcal{T}$ of $S$ in $G$ is a maximal $K$-torus. The maximal $L$-split subtorus $T$ of $\mathcal{T}$ is defined over $K$ since $\mathcal{T}$ is. If $\mathcal{T}$ does not split over $L$, then in fact, $T=S$, and $T(L)(=S(L))$ is $\Gamma$-equivariantly isomorphic to $\left(L^{\times}\right)^{r}$; where $r=L$-rank $G(=K$-rank $G)$. On the other hand, if $\mathcal{T}$ splits over $L$, then $T=\mathcal{T}$. In this case, let $a(\geq 0)$ be the number of Galois-orbits in the Tits index ( $c f$. [7]) of $G / K$ containing more than one vertex and $b$ be the number of vertices (in the Tits index) fixed under the Galois group, and $\mathfrak{L}(\subset L)$ be the splitting field of $\mathcal{T}$ if $G$ is not a triality form of type ${ }^{6} D_{4}$, and let it be a fixed cubic extension of $K$ contained in the splitting field of $\mathcal{T}$ if $G$ is a triality form of type ${ }^{6} D_{4}$. Then as $G$ is simply connected, $T(L)=\mathcal{T}(L)$ is $\Gamma$-equivariantly isomorphic to $\left(\left(\mathfrak{L} \otimes_{K} L\right)^{\times}\right)^{a} \cdot\left(L^{\times}\right)^{b}$, with $\Gamma$ acting trivially on $\mathfrak{L}$ and acting in the natural way on $L$.

Since the centralizer of $S$ in $G$ is a torus containing the torus $T$, the restriction to $S$ of any root of $G$ with respect to $T$ is nontrivial. This implies that the apartment $A$ corresponding to the maximal $K$-split torus $S$ in the building $\mathcal{B}(G / K)$, which is contained in the apartment, in the building $\mathcal{B}(G / L)$, corresponding to the maximal $L$-split torus $T$, is not contained in a wall of the latter. Let $C$ be a chamber (i.e. a simplex of maximal dimension) lying in the apartment $A$, and $\mathcal{C}$ be a chamber in the apartment corresponding to the maximal $L$-split torus $T$, in the building $\mathcal{B}(G / L)$, containing a point $x$ of $C$ in its interior. As the point $x$ is fixed under the Galois group $\Gamma, \mathcal{C}$ is $\Gamma$-stable.

TOME $129-2001-\mathrm{N}^{\mathrm{O}} 2$ 
Hence the Iwahori subgroup $I$ of $G(L)$ determined by the chamber $\mathcal{C}$ is also $\Gamma$-stable.

Let $y$ be a point of the convex subset $\mathcal{B}(G / L)^{\Gamma}$. Then the geodesic $[x, y]$ is contained in $\mathcal{B}(G / L)^{\Gamma}$. Since $x$ is an interior point of the chamber $\mathcal{C}$, the geodesic $[x, y]$ can't be contained in a wall of any apartment of the building $\mathcal{B}(G / L)$. Therefore, the points of $[x, y]$ sufficiently close to $y$, but possibly not the point $y$ itself, lie in the interior of a chamber $\mathcal{C}^{\prime}$ of the building $\mathcal{B}(G / L)$. This chamber is necessarily $\Gamma$-stable. We shall show that there is a maximal $L$-split torus $T^{\prime}, T^{\prime}$ defined over $K$ and containing a maximal $K$-split torus $S^{\prime}$, such that $\mathcal{C}^{\prime}$ lies in the apartment $A^{\prime}$ determined by $T^{\prime}$ in the building $\mathcal{B}(G / L)$.

Let $I^{\prime}$ be the Iwahori subgroup of $G(L)$ determined by $\mathcal{C}^{\prime}$. This Iwahori subgroup is also stable under $\Gamma$. Let $g \in G(L)$ be such that $I^{\prime}=g I g^{-1}$. Then for $\gamma \in \Gamma$, as $\gamma\left(I^{\prime}\right)=I^{\prime}$,

$$
c(\gamma):=g^{-1} \gamma(g)
$$

normalizes $I$ and hence it belongs to it. $\gamma \mapsto c(\gamma)$ is a $I$-valued 1-cocycle on $\Gamma$. The maximal $L$-split tori of $G$ associated with $I^{\prime}=g I g^{-1}$ (i.e. the tori such that the associated apartments contain the chamber $\mathcal{C}^{\prime}$ ) are of the form $g h T h^{-1} g^{-1}, h \in I$. We will now show that there exists an $u \in I$ such that for any $\gamma \in \Gamma$, the element

$$
(g u)^{-1} \gamma(g u)\left(=u^{-1} c(\gamma) \gamma(u)\right)
$$

belongs to $I \cap T(L)$.

Let $I^{+}$be the maximal normal pro-unipotent subgroup of $I$. Let $F$ be the residue field of $K$ ( $F$ is also the residue field of $L$ ). From our assumption that the residue field of $k$ is perfect, it follows that $F$ is algebraically closed. Now if $F$ and $K$ are of same characteristic, then the ring of integers of $K$ contains a subfield which projects isomorphically onto the residue field $F$, and if the fields $F$ and $K$ are of unequal characteristics, then the group of units of $K$ contains a canonical subgroup which projects isomorphically onto $F^{\times}$(see [5, II, Prop. 6 and 8]). From this and the explicit description of $T(L)$ given above, it is obvious that the maximal bounded subgroup $I \cap T(L)$ of $T(L)$ contains a subgroup $\Delta$ stable under the natural action of the Galois group $\Gamma$ on $T(L)$ such that $I$ is a semi-direct product $I^{+} \rtimes \Delta$ of the normal subgroup $I^{+}$and $\Delta$. For $\gamma \in \Gamma$, let

$$
c(\gamma)=g^{-1} \gamma(g)=i(\gamma) \delta(\gamma)
$$

with $i(\gamma) \in I^{+}$, and $\delta(\gamma) \in \Delta$. Then for $\gamma, \gamma^{\prime} \in \Gamma$,

$$
\begin{aligned}
c\left(\gamma \gamma^{\prime}\right) & =c(\gamma) \cdot \gamma\left(c\left(\gamma^{\prime}\right)\right) \\
& =i(\gamma) \delta(\gamma) \cdot \gamma\left(i\left(\gamma^{\prime}\right) \delta\left(\gamma^{\prime}\right)\right) \\
& =i(\gamma) \cdot \delta(\gamma) \gamma\left(i\left(\gamma^{\prime}\right)\right) \delta(\gamma)^{-1} \cdot \delta(\gamma) \gamma\left(\delta\left(\gamma^{\prime}\right)\right)
\end{aligned}
$$

BULletin DE LA SOCIÉtÉ MATHÉMATIQUE DE FRANCE 
Hence,

$\left(^{*}\right) \quad i\left(\gamma \gamma^{\prime}\right)=i(\gamma) \cdot \delta(\gamma) \gamma\left(i\left(\gamma^{\prime}\right)\right) \delta(\gamma)^{-1} \quad$ and $\quad \delta\left(\gamma \gamma^{\prime}\right)=\delta(\gamma) \gamma\left(\delta\left(\gamma^{\prime}\right)\right)$.

We define a new action of $\Gamma$ on $I^{+}$: For $\gamma \in \Gamma$ and $u \in I^{+}$, let

$$
\gamma \circ u=\delta(\gamma) \gamma(u) \delta(\gamma)^{-1}
$$

According to $\left(^{*}\right), \gamma \mapsto i(\gamma)$ is a $I^{+}$-valued 1 -cocycle on $\Gamma$ with respect to this action. The Iwahori subgroup $I$ admits a decreasing filtration by $\Gamma$-stable normal subgroups $I_{n}, n \geq 1$, converging to the trivial subgroup $\{1\}$, such that $I_{1}=I^{+}$and for all $n, I_{n} / I_{n+1}$ is a finite dimensional $F$-vector space ( $c f$. [3, $\S 2])$. Now as $L$ is a tamely ramified finite Galois extension of $K$, the Galois group $\Gamma$ is a finite group of order prime to $p$, and hence the cohomology groups $H^{1}\left(\Gamma, I_{n} / I_{n+1}\right)$ are trivial, so the cohomology set $H^{1}\left(\Gamma, I^{+}\right)$is also trivial. From this we conclude that there exists an element $u \in I^{+}$such that

$$
i(\gamma)=u(\gamma \circ u)^{-1}=u \delta(\gamma) \gamma(u)^{-1} \delta(\gamma)^{-1}
$$

Then $u^{-1} i(\gamma) \delta(\gamma) \gamma(u)=\delta(\gamma)$. Now,

$$
\begin{aligned}
(g u)^{-1} \gamma(g u) & =u^{-1} c(\gamma) \gamma(u)=u^{-1} i(\gamma) \delta(\gamma) \gamma(u) \\
& =\delta(\gamma) \quad(\in \Delta \subset T(L))
\end{aligned}
$$

Hence the maximal $L$-split torus $T^{\prime}:=g u T(g u)^{-1}$ and the subtorus $S^{\prime}:=$ $g u S(g u)^{-1}$ are defined over $K$. Also, the restriction to $T$ of the conjugation by $g u$ is defined over $K$ and so $S^{\prime}\left(\subset T^{\prime}\right)$ is a maximal $K$-split torus of $G$. Therefore, the apartment $A^{\prime}$ corresponding to $T^{\prime}$, in the building $\mathcal{B}(G / L)$, is stable under the action of the Galois group $\Gamma$ and $A^{\prime \Gamma}$ is the apartment corresponding to the maximal $K$-split torus $S^{\prime}$ in the building $\mathcal{B}(G / K)$. As $u \in I$, the apartment $A^{\prime}$ contains the chamber $\mathcal{C}^{\prime}$ and so also the point $y$. Now since $y \in A^{\prime \Gamma}$, we conclude that $y \in \mathcal{B}(G / K)$, which implies that $\mathcal{B}(G / L)^{\Gamma}=\mathcal{B}(G / K)$.

REMARK 1. - If a $k$-group $G$ is centrally $k$-isogenous to the direct product of a $k$ torus $C$ and simply connected almost $k$-simple groups $G_{i}, 1 \leq i \leq n$, and $\ell$ is a Galois extension of $k$, then the (enlarged) Bruhat-Tits building of $G / \ell$ is the product of the Bruhat-Tits buildings of $C / \ell$ and of $G_{i} / \ell, 1 \leq i \leq n$.

The building of $C / \ell$ is $X_{\ell}(C) \otimes_{\mathbb{Z}} \mathbb{R}$, where $X_{\ell}(C)$ is the free abelian group of one-parameter subgroups of $C$ defined over $\ell$. This implies at once that $\mathcal{B}(C / \ell)^{\operatorname{Gal}(\ell / k)}=\mathcal{B}(C / k)$

For a semi-simple group $\mathcal{G}$ defined over a finite separable extension $k^{\prime}$ of $k$, the Bruhat-Tits building of $R_{k^{\prime} / k}(\mathcal{G}) / \ell$ is of course the building of $\mathcal{G}\left(k^{\prime} \otimes_{k} \ell\right)$.

Using the above observations, it is easy to deduce from the result proved above that $\mathcal{B}(G / \ell)^{\operatorname{Gal}(\ell / k)}=\mathcal{B}(G / k)$ for an arbitrary connected reductive $k$ group $G$ and any finite tamely ramified Galois extension $\ell$ of $k$.

TOME $129-2001-\mathrm{N}^{\mathrm{O}} 2$ 
REMARK 2 (due to Ching-Li Chai). - Let $k$ be a field with a nontrivial discrete valuation. We assume that the field is henselian with respect to the given valuation and its residue field is perfect. For a finite extension $\ell$ of $k$, let $\hat{\ell}$ denote the completion of $\ell$. Let $G$ be a connected reductive group defined over $k$. Then for any finite extension $\ell$ of $k, G$ admits the Bruhat-Tits build$\operatorname{ing} \mathcal{B}(G / \ell)([2, \S 5])$, and the Bruhat-Tits building $\mathcal{B}(G / \hat{\ell})$ of $G / \hat{\ell}$ is equal to $\mathcal{B}(G / \ell),[4$, II, $\S 3]$. Now if $\ell$ is a tamely ramified finite Galois extension of $k$ with Galois group $\Gamma$, then $\hat{\ell} / \hat{k}$ is also a tamely ramified Galois extension whose Galois group is canonically isomorphic to $\Gamma$. As it follows from the above that $\mathcal{B}(G / \hat{\ell})^{\Gamma}=\mathcal{B}(G / \hat{k})$, we conclude that $\mathcal{B}(G / \ell)^{\Gamma}=\mathcal{B}(G / k)$. We should note here that in Rousseau's thesis, this result has been proven also when the residue field of $k$ is not perfect, and under some additional hypothesis on the reductive group $G$, if the valuation on $k$ is real but not discrete.

REMARK 3. - Let $G$ be a connected reductive group defined over a discretely valuated henselian field $k$. Let $T$ be a torus of $G$ defined and anisotropic over $k$. Let $\ell$ be the splitting field of $T$; $\ell$ is a finite Galois extension of $k$. We assume that $\ell$ is tamely ramified over $k$ and $T$ is a maximal $\ell$-split torus of $G$.

Using Rousseau's theorem established above, one can associate to $T$ a canonical point of the Bruhat-Tits building $\mathcal{B}(G / k)$ fixed under $T(k)$ as follows. Let $A$ be the apartment of the building $\mathcal{B}(G / \ell)$ corresponding to $T$. Then as $T$ is anisotropic over $k$, the Galois group $\Gamma$ of $\ell / k$ has a unique fixed point in $A$ and by Rousseau's theorem, this point actually lies in $\mathcal{B}(G / k)$.

Acknowledgement. - We thank Ching-Li Chai, Guy Rousseau, Peter Schneider and Jiu-Kang Yu for their comments on an earlier version of this note and Ching-Li Chai also for the above remark.

\section{BIBLIOGRAPHY}

[1] Borel (A.), Springer (T.A.) - Rationality properties of linear Algebraic groups II, Tohoku Math. J., 20 (1968), pp. 443-497.

[2] Bruhat (F.), Tits (J.) - Groupes réductifs sur un corps local II, Publ. Math. IHES 46, 1984.

[3] Prasad (G.), Raghunathan (M.S.) - Topological central extensions of semi-simple groups over local fields, Ann. Math. 119 (1984), pp. 143-268.

[4] Rousseau (G.) - Immeubles des groupes réductifs sur les corps locaux, Thèse Université de Paris-Sud, Orsay, 1977.

[5] Serre (J.-P.) - Local Fields, Graduate Texts in Mathematics, SpringerVerlag, New York, 1979.

[6] Steinberg (R.) - Regular elements of semi-simple groups, Publ. Math. IHES 25, 1965. 
[7] Tits (J.) - Classification of algebraic semisimple groups, Proc. AMS Symp. Pure Math. Vol. IX, 1966, pp. 33-62.

[8] Tits (J.) - Reductive groups over local fields, Proc. AMS Symp. Pure Math. Vol. XXXIII, 1979, Part 1, pp. 29-69. 